\title{
Human Hand Motion Analysis with Multisensory Information
}

\author{
Zhaojie Ju Member, IEEE, and Honghai Liu Senior Member, IEEE
}

\begin{abstract}
In order to study and analyse human hand motions which contain multimodal information, a generalised framework integrating multiple sensors is proposed and consists of modules of sensor integration, signal preprocessing, correlation study of sensory information and motion identification. Three types of sensors are integrated to simultaneously capture the finger angle trajectories, the hand contact forces and the forearm electromyography (EMG) signals. To facilitate the rapid acquisition of human hand tasks, methods to automatically synchronise and segment manipulation primitives are developed in the signal preprocessing module. Correlations of the sensory information are studied by using Empirical Copula and demonstrate there exist significant relationships between muscle signals and finger trajectories and between muscle signals and contact forces. In addition, recognising different hand grasps and manipulations based on the EMG signals is investigated by using Fuzzy Gaussian Mixture Models (FGMMs) and results of comparative experiments show FGMMs outperform Gaussian Mixture Models (GMMs) and Support Vector Machine (SVM) with a higher recognition rate. The proposed framework integrating the stateof-the-art sensor technology with the developed algorithms provides researchers a versatile and adaptable platform for human hand motion analysis and has potential applications especially in robotic hand or prosthetic hand control and Human Computer Interaction (HCI).
\end{abstract}

Index Terms-Multisensory Information, Hand Motion Analysis, Data Glove, Contact Force, EMG

\section{INTRODUCTION}

$\mathbf{T}$ HE human hand contains 27 bones with roughly 25 degrees of freedom, which are driven by 17 intrinsic muscles in the hand itself and 18 extrinsic muscles in the forearm. Such a highly articulated and complex system is capable of performing more complicated and dexterous tasks than any other existing systems, and it has been regarded as a rich source of inspiration for the engineers and scientists to design human-like robotic and prosthetic hands and to learn and model human hand motion skills. Human hand motion analysis is attracting engagement of more and more researchers in the areas of neuroscience, biomedical engineering, robotics, human-computer interaction (HCI) and Artificial Intelligence (AI).

Z. Ju is with the Intelligent Systems \& Biomedical Robotics Group, School of Creative Technologies, University of Portsmouth PO1 2DJ, UK. Email: zhaojie.ju@port.ac.uk

H. Liu is with the Intelligent Systems \& Biomedical Robotics Group, School of Creative Technologies, University of Portsmouth PO1 2DJ, UK. Email: honghai.liu@port.ac.uk (corresponding author)

The authors would like to acknowledge financial support from UK National Engineering and Physical Scientific Research Council under Grant No. EP/G041377/1, from the Royal Society Grant under grant No. IJP08/R2 and from British Council for an Overseas Research Scholarship Award.
Different properties involved in the human hand motions provide people with rich sensory information such as hand position, velocity, force and their changes with the time to build up computational models of these motions. Hand motion capturing systems can be mainly categorized into different ways: data glove based capturing, markers based capturing, vision based capturing, haptics based capturing and Electromyography (EMG) based capturing. Data glove and markers are preferred and have been widely adopted [1], [2], [3] because they use highly precise sensors to achieve hand dynamic gestures including positions, velocities and accelerations. Vision based capturing is becoming more and more popular mainly due to advances in computer vision algorithms to solve the ill-posed problem of 3D construction out of 2D images [4], [5], [6]. Compared to data gloves, using cameras to capture the hand motions is a more natural and non-contact way, which does not cling to hands and does not limit movements and volumes of the finger activities. However, the vision sensor requires quality image processing techniques and suffers from limitations of the low accuracy and high computational cost. Recently, commercially available depth cameras such as Kinect have recently been successfully employed to extract robust human body skeleton especially for games. They have also been applied to the sign language and hand gesture recognition [7], [8]. However, it is still challenging to extract the skeleton of the hand, since the hand has a small shape and high degrees of freedom compared to the main body. Haptics/tactile/force information is an important property of the human hand motions, especially for the object manipulations [9], [10], [11], [12], e.g., Park et al. [13] designed a haptic interaction system for transferring expertise in teleoperation-based manipulation between two human users and demonstrated that the haptic knowledge is transferable both through the haptic force guidance as well as through the robotic demonstration. EMG signal is the electrical potential generated by muscle contractions and is very useful to study human movements. Different from all above sensory information capturing techniques, EMG signals for analysing human hand motions are usually taken from the human forearm instead of the hand itself, since the majority of the finger movements are driven by the extrinsic muscles in the forearm and it can be used to indirect estimate manipulation force a human hand applies [14], [15]. It is the most natural and promising way to control the prosthetic hands and attracting more and more researchers to use such signals to analyses human hand motions for prosthetic hands control [16], [17], [18], HCI [19], [20] and health recovery [21], [22].

Integration of the above multiple sensory information is 
essential for the human hand motion analysis. Ceruti et al. [23] integrated data glove with haptics sensors to capture both the finger angles and the finger tip forces and Norman et al. [24] employed the data glove with both finger position and contact force information to analyse human in-hand manipulation. Romano et al. [25] introduced the SlipGlove providing tactile cues associated with slip between the glove and a contact surface. Such gloves with haptic-IO capability provide the vital information of human hand motion and greatly enhance the capturing of human hand skills. Yang et al. [26] proposed an on-line estimation method for the hand grasp force based on the surface EMG extracted from human forearm synchronously. More examples could be found in [27], which presents a comprehensive review of recent scientific findings and technologies including human hand analysis and synthesis, hand motion capture, recognition algorithms and applications. However, to the best of our knowledge, there are few studies, which have integrated the muscle signals with the contact forces and hand finger trajectories for human hand motion analysis.

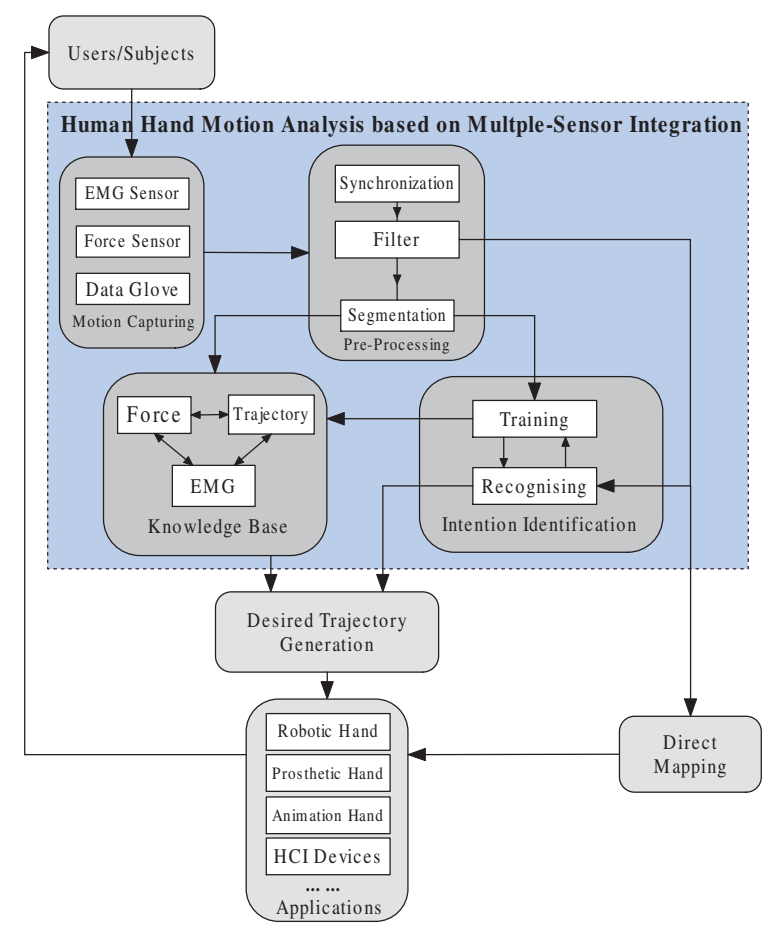

Fig. 1. Framework of Multiple-sensor Integration for Human Hand Motion Analysis

In this paper, we propose a framework of multiple-sensor integration of the data glove, force sensors and EMG sensors for human hand motion analysis, as shown in Fig. 1 based on our previous study [28]. The framework consists of four main components, i.e. motion capturing module, preprocessing module, knowledge base module, and motion identification module. The motion capturing module is to use different types of sensors to transfer the sensory information into digital signal recognisable to computers. In this paper, three types of sensors are used, including CyberGlove joint angle sensors, FingerTPS pressure sensors and Trigno wireless EMG sensors, which capture the hand gestures, contact forces and muscle contraction signals from various hand motions respectively. The preprocessing module is to synchronise and filter the original digital data and segment them into individual tasks. Automatic synchronisation and segmentation are investigated to improve the efficiency of the acquisition of human hand tasks. The knowledge base module stores the human hand motion primitives, manipulation scenarios and correlations among the different sensory information. In the knowledge base module, we investigate the correlations of the finger trajectories, contact forces and EMG signals. The identification module is to use the clustering and machine learning methods to train the motion models using the preprocessed datasets and to recognise new or testing data from one or more types of sensors. Different recognition methods including Fuzzy Gaussian Mixture Models (FGMMs), Gaussian Mixture Models (GMMs) and Support Vector Machine (SVM) are investigated for the EMG based manipulation identification and comparison results are analysed. In addition, the desired trajectory generation module can be regarded as the link between the motion analysis framework and specific applications. It generates the desired trajectory based on the results of the human motion analysis framework for applications such as controlling robotic hand and prosthetic hand, planning animation hand motion and $\mathrm{HCI}, e . g$. adapting motion trajectories into artificial hands [29]. The desired trajectory module is not the main focus of this paper and will be one part of the future work. This paper is structured as follows: The multiple sensor capture system and the preprocessing are described in Section II. The studies of the correlations of EMG, forces and finger trajectories in the human hand motion knowledge based module have been given in Section III. The motion training and recognition based on EMG signals have been investigated in Section IV. Finally, Section V concludes the paper with remarks.

\section{Multiple-Sensor Hand Motion Capture System}

In order to capture the finger trajectories, the contact force signals and the muscle signals, the multiple-sensor hand motion capture system consists of a high-accuracy finger jointangle measurement system which is CyberGlove, a wireless tactile force measurement system from FingerTPS and a highfrequency EMG capture system with Trigno Wireless Sensors. In this section, the system configuration and the preprocessing module including the hardware based synchronisation and segmentation will be described, followed by the data capturing at the end.

\section{A. System Configuration}

As a fully instrumented glove, the CyberGlove shown in Fig 2.(a) uses proprietary resistive bend-sensing technology to accurately transform hand and finger motions into real-time digital joint-angle data and provides up to 22 high-accuracy joint-angle measurements, where three flexion sensors per finger, four abduction sensors, a palm-arch sensor, and sensors to measure flexion and abduction. Tracking sensors are also included in the CyberGlove system to measure the position and orientation of the forearm in space. The sensor resolution 


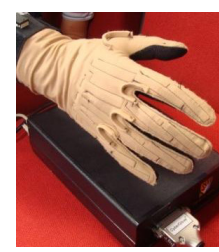

(a)

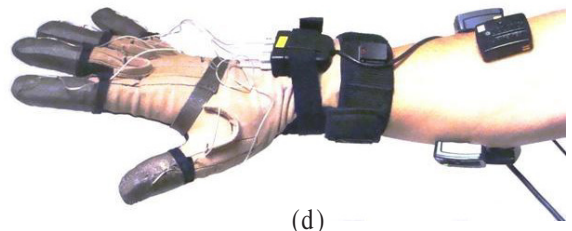

(d)

Fig. 2. Sensors: (a) CyberGlove; (b) FingerTPS Force Sensor; (c) Trigno Wireless EMG Sensor; (d) Multiple Sensors for One Hand

is 0.5 degrees, the repeatability is one degree, and the sampling rate is $150 \mathrm{~Hz}$. Highly sensitive capacitive-based pressure sensors of FingerTPS shown in Fig 2.(b) have also been utilised to reliably quantify forces applied by the human hand. Calibration is achieved by using a reference force sensor. The system has 6 comfortable capacitive sensors per hand, wirelessly connected to the computer. The sensors have a data rate of $40 \mathrm{~Hz}$. The full scale range is 10-50 lbs and the sensitivity is $0.01 \mathrm{lbs}$. Video images can be captured and displayed in real-time, synchronised with tactile data. In addition, the EMG capture system employs Trigno Wireless Sensors shown in Fig 2.(c) and has 16 EMG channels and 48 accelerometer channels. The resolution is 16 bits and the sampling rate is $4000 \mathrm{~Hz}$. Its size is $37 \mathrm{~mm} \times 26 \mathrm{~mm} \times$ $15 \mathrm{~mm}$ and the range of its guaranteed performance is 40 meters. It has 64 channels of real-time analog output for motion capture integration. Trixial accelerometers can be used to resolve orientation with respect to the normal force, as well as capture dynamic movements and impacts. These data are captured simultaneously with the EMG data.

\section{B. Synchronization}

The integration of the data glove, the force sensors and the EMG sensors requires a high-speed digital signal processor (DSP) to acquire, process and send raw synchronised information digitally to a PC for analysis shown in Fig. 3. The CPU speed of the DSP is greater than $10 \mathrm{MHz}$ for a faster and efficient data acquisition. The force signals from FingerTPS and the EMG signals from the Trigno system are sampled simultaneously at $4 \mathrm{~K}$ samples per second by the onboard Analog to Digital Converter (ADC). The data captured from the CyberGlove can be gathered via a universal asynchronous receiver/transmitter (UART) and transmit back to a computer along with other data. The interface connection between the DSP and the computer is USB whose maximum data transfer rate is 10 megabits per second. The three devices are sampled simultaneously and the resolution is 16 bits.

\section{Segmentation}

To separate each motion with the next motion in the same type, before moving the hand, the hand should be in

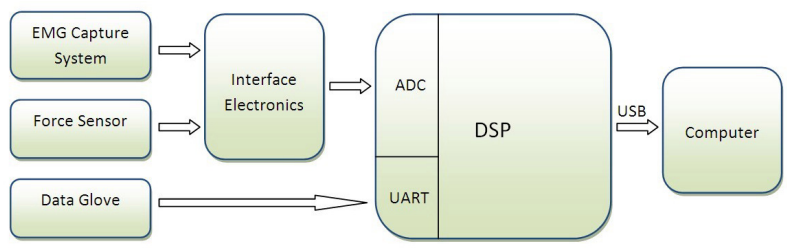

Fig. 3. Hardware based synchronization of the data glove, the force sensor and the EMG capture system.

intermediate state that is a flat hand with no strength. It is assumed that the motion begins when the finger angles change from the intermediate state, and ends when the finger angles change to the intermediate state. In this way, the computer can identify both the start point and end point of each motion using threshold based methods, shown in Fig 4. Five-quick-

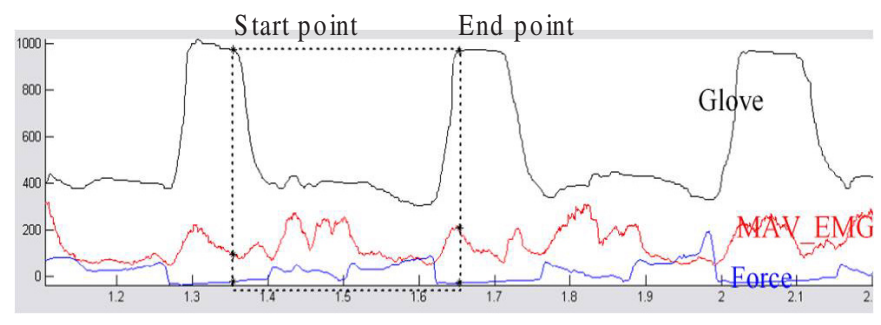

Fig. 4. Start and end point for the motion separation

grasp is to do five power grasps quickly and it enables the muscle to quickly continuously contract five times, and enables the sensory signals with five prominent maximum in their trajectories, simultaneously, shown in Fig. 5. These five contractions and maximum are very easy to be identified using peak-detection algorithms. To automatically segment the motions, five-quick-grasp is utilised in the experiments when one type of the motions is finished and the participants are performing the next type of the motions. During the experiment, even trained participants may have difficulties in fulfilling all the tasks at one go. Usually, the motions required in the experiment cannot be finished properly; for example, the cup is dropped by accident. In this case, the data recorded previously is invalid and the experiment needs to be repeated. To solve this problem, we design a protocol that if the motion fails, the participants need to do a four-quick-grasp that indicates the motion recorded before is invalid. In the separation process, the motion before the 'four-quick-grasp' will be deleted automatically.

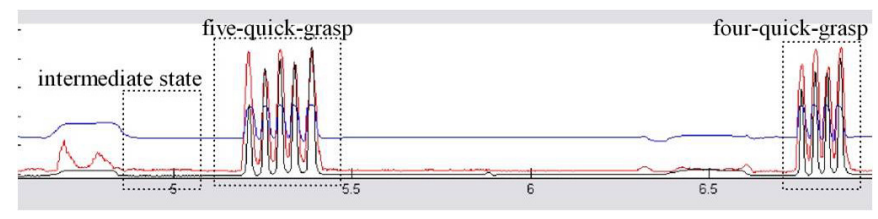

Fig. 5. Intermediate State, Five-quick-grasp and Four-quick-grasp 


\section{Data Capturing}

The sEMG of 5 forearm muscles shown in Figure 6, i.e. flexor carpi radialis, flexor carpi ulnaris, flexor pollicis longus, flexor digitorum profundus and extensor digitorum, were measured. To obtain clearer signals, subjects were scrubbed with alcohol and shaved if necessary and then electrodes were applied over the body using the die cut medical grade doublesided adhesive tape. Electrodes locations were selected according to the musculoskelet of these five muscles and confirmed by muscle specific contractions, which include manually resisted finger flexion, extension and abduction. The real time sEMG signals were visualised on a computer screen giving participants feedback to choose the positions of electrodes with stronger sEMG signals. During the experiments, the accelerometers of the EMG systems and the position tracking of the CyberGlove have not been used.

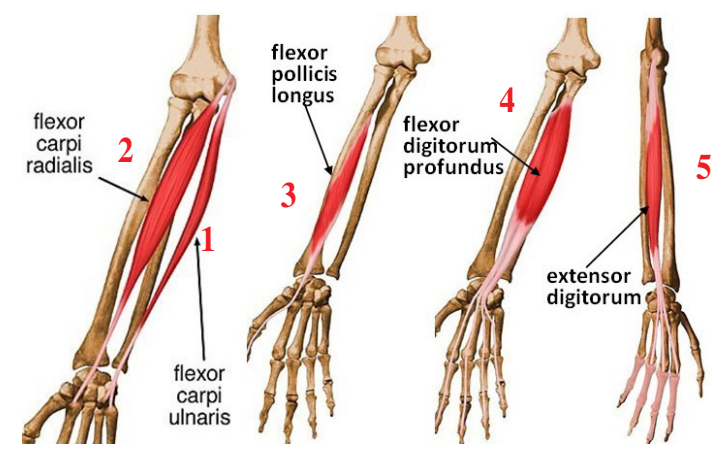

Fig. 6. Muscle positions

Eight healthy right-handed subjects including 2 females and 6 males volunteered for the study. Their ages range from 23 to 40 and average is 32.5 years; body height average is $175.5 \mathrm{~cm}$; body mass average is $70 \mathrm{~kg}$. All participants gave informed consent prior to the experiments and the ethical approval for the study was obtained from University of Portsmouth CCI Faculty Ethics Committee. All subjects were trained to manipulate different objects. Participants had to perform ten

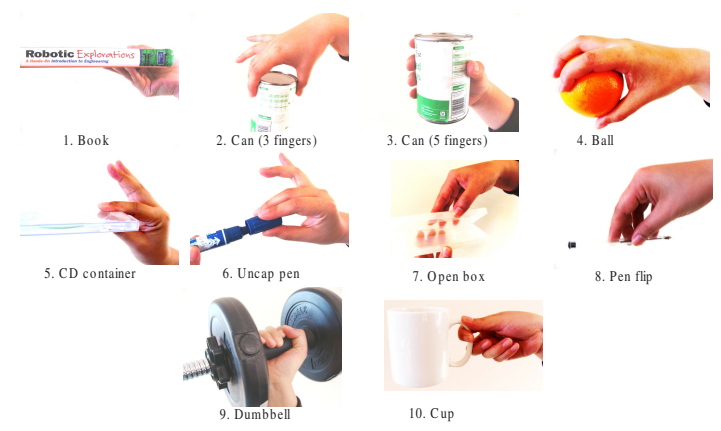

Fig. 7. Hand motions including grasps and in-hand manipulations

grasps or in-hand manipulations which are shown in figure 7 and the motions are listed as following

1) Grasp and lift a book using five fingers with the thumb abduction.

2) Grasp and lift a can full of rice using thumb, index finger and middle finger only.
3) Grasp and lift a can full of rice using five fingers with the thumb abduction.

4) Grasp and lift a big ball using five fingers.

5) Grasp and lift a disc container using thumb and index finger only.

6) Uncap and cap a marker pen using thumb, index finger and middle finger.

7) Open and close a pen box using five fingers.

8) Pick up a pencil using five fingers, flip it and place it on the table.

9) Hold and lift a dumbbell.

10) Grasp and lift a cup using thumb, index finger and middle finger.

The way to grasp or manipulate objects had been shown to the participants in the demonstration before they performed and every motion lasted about 2 to 4 seconds. Each motion was repeated 10 times. Between every two repetitions, participants had to relax the hands for 2 seconds in the intermediate state that is opening hand naturally without any muscle contraction. These intermediate states were used to segment the motions. Once one motion with ten repetitions was finished, participants had to relax the hand for 2 minutes before the next motion started. This was designed to overcome the effects of muscle fatigue.

\section{Correlations of Finger Trajectories, Contact FORCES AND THE EMG SIGNALS}

The hand motion capture system integrating multiple sensors provides us with the ability to study the correlations among the finger force, the finger trajectory and the muscle signal. Spearman's rank correlation coefficient or Spearman's rho is a non-parametric measure of statistical dependence between two variables, and it assesses how well the relationship between two variables can be described using a monotonic function [30]. In this section, we will concentrate on Spearman's rho which is best-known in economic and social statistics. Copula is a popular statistical tool to model and estimate the distribution of random vectors by estimating marginals and to describe the dependence between random variables. The copula of a random vector can capture the properties of the joint distribution which are invariant under transformations of the univariate margins [31], so it is natural to consider the dependence measure, Spearman's rho, which is based on the distribution's copula [32]. Sklar's theorem is fundamental to the theory of copula and underlies most applications of the copula. It elucidates the role that copula plays in the relationship between multivariate distribution functions and their univariate margins. More details of the copula definition and Sklar's theorem can be found in [33], [34]. In this section, we will investigate correlations of the sensory information using the Spearman's rho based on the empirical copula.

\section{A. Empirical Copula and Dependence Estimation}

The empirical copula is a characterisation of the dependence function between variables based on observational data using order statistics theory and it can reproduce any pattern found in 
the observed data. If the marginal distributions are normalised, the empirical copula is the empirical distribution function for the joint distribution.

Definition III-A.1. Let $\left\{\left(x_{k}, y_{k}\right)\right\}_{k=1}^{n}$ denote a sample of size $n$ from a continuous bivariate distribution. The empirical copula [33] is the function $C_{n}$ given by

$$
C_{n}\left(\frac{i}{n}, \frac{j}{n}\right)=\frac{\operatorname{Num}\left((x, y) \mid x \leq x_{(i)}, y \leq y_{(j)}\right)}{n}
$$

where $i, j$ are variables in the copula function, $1 \leq i, j \leq$ $n ; \operatorname{Num}\left((x, y) \mid x \leq x_{(i)}, y \leq y_{(j)}\right)$ returns the number of the pairs of $(x, y)$ which satisfy the condition $x \leq x_{(i)}$ and $y \leq$ $y_{(i)} ; x_{(i)}$ and $y_{(j)}$, denote order statistics from the sample.

The empirical copula frequency $c_{n}$ is given by

$$
c_{n}\left(\frac{i}{n}, \frac{j}{n}\right)=\left\{\begin{array}{c}
1 / n, i f\left(x_{(i)}, y_{(j)}\right) \subset(x, y) \\
0, \text { otherwise }
\end{array}\right.
$$

Note that $C_{n}$ and $c_{n}$ are related via

$$
C_{n}\left(\frac{i}{n}, \frac{j}{n}\right)=\sum_{p=1}^{i} \sum_{q=1}^{j} c_{n}\left(\frac{p}{n}, \frac{q}{n}\right)
$$

Theorem III-A.1. Let $C_{n}$ denotes the empirical copula for the sample $\left\{\left(x_{k}, y_{k}\right)_{k=1}^{n}\right\}$. If $\rho$ denotes the sample versions of Spearman's rho [35], [36], then

$$
\rho=\frac{12}{n^{2}-1} \sum_{i=1}^{n} \sum_{j=1}^{n}\left[C_{n}\left(\frac{i}{n} \cdot \frac{j}{n}\right)-\frac{i}{n} \cdot \frac{j}{n}\right]
$$

As a non-parametric measure of statistical dependence between two variables, Spearman's rho can be used to assess how well the relationship between two variables can be described using a monotonic function. According to the definition and theorem, we can estimate correlations between variables using empirical copula and Spearman's rho.

\section{B. Experimental Results}

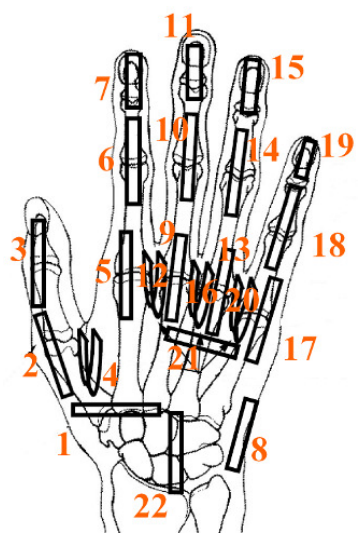

Fig. 8. Sensor positions in CyberGlove

In this section, correlations among Mean Absolute Value (MAV) of sEMG signal, finger force and finger angle trajectory have accessed by the Spearman's rho calculated based on the copula. A sliding window with size of $300 \mathrm{~ms}$ and increment of 50ms has been used to compute MAV. Fig. 6 and Fig. 8
TABLE II

RELATION BETWEEN THE MUSCLE CONTRACTION AND THE FINGER TIP FORCE OF THE MOTION EIGHT; THE ROW INDEXES 1-5 REPRESENT THE DIFFERENT EMG SENSORS SHOWN IN FIG. 6; THE COLUMN INDEXES 1-5 REPRESENT THE DIFFERENT FORCE SENSORS; THE RELATIVE STRONG RELATIONS FOR EACH EMG SENSOR ARE HIGHLIGHTED

\begin{tabular}{|l|l|l|l|l|l|l|}
\hline & thumb & index & middle & ring & little & palm \\
\hline 1 & .32 & .26 & .28 & $\mathbf{. 5 6}$ & $\mathbf{. 6 2}$ & -.14 \\
& $(.08)$ & $(.09)$ & $(.10)$ & $\mathbf{( . 0 7 )}$ & $\mathbf{( . 0 9 )}$ & $(.06)$ \\
\hline 2 & .34 & $\mathbf{. 4 1}$ & $\mathbf{. 5 6}$ & .16 & .17 & .16 \\
& $(.09)$ & $\mathbf{( . 0 8 )}$ & $\mathbf{( . 0 6 )}$ & $(.07)$ & $(.07)$ & $(.08)$ \\
\hline 3 & $\mathbf{. 7 3}$ & .45 & .32 & .22 & .33 & -.12 \\
& $\mathbf{( . 0 9 )}$ & $(.10)$ & $(.09)$ & $(.06)$ & $(.11)$ & $(.06)$ \\
\hline 4 & .33 & $\mathbf{. 4 5}$ & $\mathbf{. 4 2}$ & .21 & .32 & .02 \\
& $(.09)$ & $\mathbf{( . 0 7 )}$ & $\mathbf{( . 0 6 )}$ & $(.05)$ & $(.07)$ & $(.11)$ \\
\hline 5 & .15 & $\mathbf{- . 4 2}$ & $\mathbf{- . 4 6}$ & -.16 & -.25 & .09 \\
& $(.10)$ & $\mathbf{( . 0 8 )}$ & $\mathbf{( . 0 8 )}$ & $(.07)$ & $(.10)$ & $(.09)$ \\
\hline
\end{tabular}

present position indexes of the EMG sensors and CyberGlove sensors respectively. The index of the force sensors are one to six for thumb, index finger, middle finger, ring finger, small finger and palm respectively, shown in Fig. 2 (b). Table I shows the average correlation coefficients (Spearman's rho) and their variances in the bracket for the relations between the muscle contractions and the finger angle trajectories of the motion eight, which is picking up a pencil and flipping it by eight different subjects. From the table, it is clear that there are significant relationships between the muscle signals and the finger angle trajectories: the first muscle signal captured from the flexor carpi ulnaris has strong positive relationships with the little finger and ring finger movements; for the second muscle signal, the contraction from the flexor carpi radialis has the strongest positive relationship with the index finger movements and the second strongest the middle finger movements; the third muscle signal from the flexor pollicis longus has the strongest positive relationship with the thumb movement; similar to the second muscle signal, the fourth muscle signal from the flexor digtorum profundus has the strongest relationship with the index finger and the middle finger; the fifth muscle signal from extensor digitorum has strong inverse relationship with the index finger and the middle finger. For each muscle signal, the strongest correlations are highlighted in the table. In addition, from the correlation coefficients for the sensor number 4, 8, 1216,20 and 22 which are measuring the finger adduction, abduction and the angles on the palm and wrist, it is hard to see their obvious relationships with the muscle signals compared to the flexion and extension angles such as thumb fingers in the table. Though the third muscle signal has 'large' relationship with the sensor number 4, 12 and 16, their spearman's rho values are much smaller than those between the thumb finger and the third muscle signal.

On the other hand, the relationships between the muscle signals and the finger tip forces have also been studied and the results are shown in the Table II. From the table, the results are consistent with the results in the Table I except that all the correlation coefficient averages and variances are smaller than those in the Table I. 
TABLE I

RELATION BETWEEN THE MUSCLE CONTRACTION AND THE FINGER ANGLE TRAJECTORIES OF THE MOTION EIGHT; THE ROW INDEXES 1-5 REPRESENT THE DIFFERENT EMG SENSORS SHOWN IN FIG. 6; THE COLUMN INDEXES 1-22 REPRESENT THE DIFFERENT CYBERGLOVE SENSORS SHOWN IN FIG. 8; THE RELATIVE STRONG RELATIONS FOR EACH EMG SENSOR ARE HIGHLIGHTED

\begin{tabular}{|c|c|c|c|c|c|c|c|c|c|c|c|}
\hline & \multicolumn{3}{|c|}{ Thumb Finger } & & \multicolumn{3}{|c|}{ Index Finger } & & \multicolumn{3}{|c|}{ Middle Finger } \\
\hline & 1 & 2 & 3 & 4 & 5 & 6 & 7 & 8 & 9 & 10 & 11 \\
\hline 1 & $.14(.06)$ & $.23(.07)$ & $-.31(.06)$ & $-.25(.04)$ & $.11(.06)$ & $-.10(.06)$ & $.17(.04)$ & $.24(.04)$ & $.25(.06)$ & $.39(.06)$ & $.29(.07)$ \\
\hline 2 & $.42(.09)$ & $-.34(.07)$ & $.04(.06)$ & $-.32(.07)$ & $.55(.10)$ & $.41(.05)$ & $.49(.05)$ & $.21(.04)$ & $.81(.09)$ & $.48(.07)$ & $.47(.08)$ \\
\hline 3 & $.73(.04)$ & $.68(.12)$ & $.71(.07)$ & $-.52(.04)$ & $.33(.03)$ & $.55(.10)$ & .48(.09) & $.05(.03)$ & $.43(.07)$ & $.39(.08)$ & $.31(.04)$ \\
\hline 4 & $.00(.05)$ & $.17(.07)$ & $.28(.08)$ & $.11(.06)$ & $.68(.05)$ & $.45(.06)$ & $.36(.08)$ & $.44(.05)$ & $.52(.09)$ & $.44(.05)$ & $.31(.04)$ \\
\hline 5 & $-.19(.11)$ & $.13(.07)$ & $-.10(.08)$ & $-.12(.09)$ & $-.42(.11)$ & $-.31(.05)$ & $-.25(.06)$ & $-.13(.07)$ & $-.43(.09)$ & $-.21(.10)$ & $-.14(.12)$ \\
\hline & & \multicolumn{3}{|c|}{ Ring Finger } & & \multicolumn{3}{|c|}{ Little Finger } & & & \\
\hline & 12 & 13 & 14 & 15 & 16 & 17 & 18 & 19 & 20 & 21 & 22 \\
\hline 1 & $-.18(.06)$ & $.63(.06)$ & $.51(.06)$ & $.40(.06)$ & $.23(.05)$ & $.83(.06)$ & $.49(.06)$ & $.48(.07)$ & $.22(.04)$ & $-.06(.08)$ & $-.37(.08)$ \\
\hline 2 & $.32(.08)$ & $-.07(.06)$ & $-.37(.09)$ & $-.38(.05)$ & $.26(.06)$ & $-.21(.08)$ & $-.36(.09)$ & $-.25(.07)$ & $-.17(.05)$ & $-.11(.08)$ & $-.43(.07)$ \\
\hline 3 & $.53(.05)$ & $.18(.08)$ & $.43(.03)$ & $.51(.05)$ & $.51(.05)$ & $.21(.06)$ & $.48(.03)$ & $.52(.08)$ & $.31(.07)$ & $.19(.10)$ & $.10(.10)$ \\
\hline 4 & $.21(.06)$ & $.30(.08)$ & $.01(.05)$ & $.19(.05)$ & $-.06(.06)$ & $.32(.09)$ & $.16(.05)$ & $.25(.07)$ & $.24(.08)$ & $.28(.08)$ & $-.16(.06)$ \\
\hline 5 & $-.08(.10)$ & $.25(.06)$ & $-.02(.13)$ & $.04(.09)$ & $-.21(.09)$ & $.22(.10)$ & $-.14(.11)$ & $-.13(.09)$ & $-.24(.06)$ & $-.11(.08)$ & $.16(.08)$ \\
\hline
\end{tabular}

\section{Motion Recognition Via EMG Intention}

As one of the most active research areas, hand motion recognition, in general, consists of neural network approaches [37], support vector machines (SVM) methods [38], [39], rule-based reasoning approaches [40] and probabilistic graphical models [41], [3]. Neural networks are more efficient and achieve better results for complex applications with a huge amount of data. SVMs are very popular because the optimisation problem has a unique solution, but the choice of the kernel function has a significant effect on its performance and the best choice is application dependent. The rule-based reasoning approaches are easy to implement but their performance highly depends on the applications. Probabilistic graphical models such as hidden Markov models (HMM) and Gaussian Mixture Models (GMMs) have demonstrated their high potential in hand gesture recognition, since they are very rich in mathematical structure and hence their theoretical basis can be adopted for a wide range of applications. Gaussian Mixture Models (GMMs) are one of the most statistically mature methods in pattern recognition and machine learning [41], [42], and has been successfully implemented to identify the high frequency signals such as in speech and EMG recognition [43], [44]. In our previous work [45], Fuzzy Gaussian Mixture Models (FGMMs) are proposed with a better fitting performance and a faster convergence speed than conventional GMMs. In this paper, we will employ FGMMs to recognise hand motions including different hand grasps and in-hand manipulations via the EMG based signals. In this section, we will firstly revisit the Expectation-Maximization (EM) algorithm for the FGMMs and then propose the novel training and recognising methods. The comparative experimental results and discussions are given in the end.

\section{A. Fuzzy Gaussian Mixture Models}

The distance based FGMMs are chosen and referred as FGMMs in this paper, since its performance is better than the probability based FGMMs [45]. The processing of training with FGMMs is summarised as follows.

Let $\mathcal{X}=\left\{x_{1}, x_{2}, \ldots, x_{n}\right\}$ be the $d$ dimensional observed dataset with $n$ samples; $k \geq 2$ be the number of the components; $n$ be the number of the sampling points; $m>1$ be the degree of fuzziness; $\varepsilon>0$ be a small preset real positive number. The initialisation of FGMMs is achieved by Fuzzy CMeans (FCM). The iteration of EM algorithm for the FGMMs shows:

- E-Step: Compute 'expected' classes of all data points for each class.

$$
u_{i t}=\left[\sum_{j=1}^{k}\left(\frac{d_{i t}}{d_{j t}}\right)^{\frac{2}{m-1}}\right]^{-1}
$$

where $u_{i t}$ is the degree of membership of $x_{t}$ in the $i$ th cluster; $d_{i t}$ is the dissimilarity between point $x_{t}$ and $i$ th cluster, which can be archived by

$$
\left\{\begin{array}{cc}
\frac{\exp \left(\frac{\left(x_{t}-\mu_{i}\right)^{T} \Sigma_{i}^{-1}\left(x_{t}-\mu_{i}\right)}{2}\right)}{\left(\alpha_{\mathrm{i}}(2 \pi)^{-\frac{\mathrm{d}}{2}}\left|\Sigma_{i}\right|^{-\frac{1}{2}}\right)^{\frac{m-1}{m}}} & \left(\left|a_{i}\right|<\varepsilon\right) \\
\frac{1}{\alpha_{i}^{\frac{m-1}{m}} p_{i}\left(x_{t} \mid \theta_{i}\right)} & \left(\left|a_{i}\right| \geq \varepsilon\right)
\end{array}\right.
$$

where $\mu_{i}$ is the mean and $\Sigma_{i}$ is the covariance matrix of the $i$ th Gaussian component; $\alpha_{i}$ is the weight of $i$ th component; $a$ is first parameter of the standard $y=a x^{2}+$ $b$ which is used to shape the principle component axis; $p_{i}\left(x_{t} \mid \theta_{i}\right)$ is the probability density function of point $x_{t}$ to the $i$ th component and it has:

$$
\begin{aligned}
& p\left(x_{t} \mid \theta\right)= \\
& \left(\sum_{j=1}^{J_{t}} \prod_{s=1}^{2} \frac{\exp \left(\frac{-l_{j}^{2}\left(v_{s t}\right) m}{2 \Sigma_{s}(m-1)}\right)}{\sqrt{2 \pi\left|\Sigma_{s}\right|}} \prod_{s=3}^{d} \frac{\exp \left(\frac{-v_{s t}^{2} m}{2 \Sigma_{s}(m-1)}\right)}{\sqrt{2 \pi\left|\Sigma_{s}\right|}}\right)^{\frac{m-1}{m}}
\end{aligned}
$$

where $l_{j}\left(v_{1 t}\right)$ is the arc length of the $j$ th projected coordinate $z=\left[z_{1 j}, z_{2 j}\right]$, which is transferred from point $x_{t}$, on the standard curve principle axis; $l_{j}\left(v_{2 t}\right)$ is the distance between the transferred point $\left[v_{1 t}, v_{2 t}\right]$ and its projected point $z$. More details about how to get these projected points or transferred points can ben found in the appendix of this paper.

- M-Step: Compute Maximum likelihood given the data's class membership distributions.

If $\left|a_{i}\right|<\varepsilon$ 


$$
\begin{gathered}
\mu_{i}^{n e w}=\frac{\sum_{t=1}^{n} u_{i t}^{m} x_{t}}{\sum_{t=1}^{n} u_{i t}^{m}} \\
\sum_{i}^{n e w}=\frac{\sum_{t=1}^{n} u_{i t}^{m}\left(x_{t}-\bar{\mu}_{i}\right)\left(x_{t}-\bar{\mu}_{i}\right)^{T}}{\sum_{t=1}^{n} u_{i t}^{m}}
\end{gathered}
$$

If $|a| \geq \varepsilon$

$$
\left(C_{i}^{\text {new }}, T_{i}^{\text {new }}, Q_{i}^{\text {new }}\right)=\operatorname{LSFM}\left(P C A\left(\mathcal{X} \cdot U_{i}\right)\right)
$$

$U_{i}=\left[u_{i 1}, \ldots, u_{i n}\right] ; P C A()$ is the principal component analysis function for estimating the translation matrix $T_{i}^{\text {new }}$ and rotation matrix $U_{i}^{\text {new }} . \operatorname{LSFM}()$ is leastsquares fitting method for estimating control parameters $C_{i}^{\text {new }}=(a, c)$ which shapes the curve axis with standard curve $y=a x^{2}+b$; and the new estimated mean and covariance are:

$$
\begin{gathered}
\mu_{i}^{n e w}= \\
\frac{\sum_{t=1}^{n} u_{i t}^{m} x_{t}}{\sum_{t=1}^{n} u_{i t}^{m}}+\left(Q_{i}^{n e w}\right)^{-1} \underbrace{[0, b, 0, \cdots, 0]^{T}}_{d}+T_{i}^{n e w} \\
\sum_{i e}^{n e w}=\frac{\sum_{t=1}^{n} u_{i t}^{m} \bar{L}_{t e}^{(i)}}{\sum_{t=1}^{n} u_{i t}^{m}} \quad(e=1,2) \\
\sum_{i(3-d)}^{n e w}=\frac{\sum_{t=1}^{n} u_{i t}^{m}\left(x_{t}-\mu_{i}^{n e w}\right)_{(3-d)}\left(x_{t}-\mu_{i}^{n e w}\right)_{(3-d)}^{T}}{\sum_{t=1}^{n} u_{i t}^{m}}
\end{gathered}
$$

Details of the EM algorithm for FGMM can be found in the appendix B.

\section{B. Training Models via FGMMs}

Root Mean Square(RMS), modelled as amplitude modulated Gaussian random process, relates to the constant force and non-fatiguing contraction. Suppose the EMG signal is $f(t)$, where $1 \leq t \leq N, N$ is the number of the sample points, then the RMS is given by

$$
f_{r m s}(t)=\sqrt{\frac{1}{2 w+1} \sum_{i=t-w}^{t+w} f^{2}(i)}
$$

where $2 w+1$ denotes the length of the signal window, and $1 \leq i \leq N$. In this paper, we use RMS as the EMG feature, since RMS shows powerful performance in robust noise tolerance than other time domain features such as integrated EMG, simple square integral, mean absolute value, mean absolute value slop, and variance [46]. The RMS feature is used both for the FGMMs learning and recognising.

The inputs of the FGMMs include the RMS feature which in our case is a five dimensional time series, number of components $k$, degree of fuzziness $m$ and threshold $\varepsilon$, which have been discussed in Section IV-A. Then EM algorithm for FGMMs has been utilised to find the optimised centres of the components $\mu$, their covariances $\Sigma$ and the control parameters $C, T, Q$, which are the outputs of the FGMMs and will be used in the recognition process. For the details to implement the EM algorithm of FGMMs, please refer to the algorithm appendix in [45]. An example of the model trained by the introduced FGMMs with six components on the extracted RMS is shown in Fig. 9.

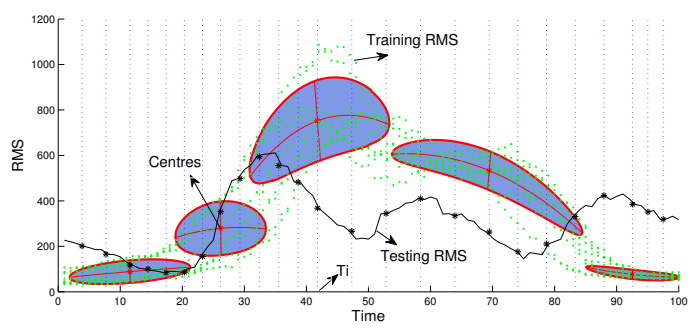

Fig. 9. An example of the FGMMs trained result with six components (blue) on the RMS feature(greed dots); The black line is the RMS from the testing motion; black dots are the re-sampled points for the testing RMS at $T_{i}$ time instance

\section{Recognition}

Supposing there are $k$ components in the FGMMs trained result. To recognise the testing motion, similarity function is proposed in Equ. 15. The similarity of the testing motion and the trained model of FGMMs is defined by the normalised log-likelihood between the re-sampled testing points and the FGMMs components as:

$$
S i=\frac{1}{5 k} \sum_{j=1}^{5 k} \log \left(\sum_{i=1}^{k} \alpha_{i} p_{i}\left(x_{T_{j}} \mid \theta_{i}\right)\right)
$$

where $\alpha_{i}$ is the mixing coefcient of the $i$ th component, if the component's curvature parameter $a_{i}<\varepsilon$, the $p(x \mid \theta)$ will be calculated by:

$$
p(x \mid \theta)=\frac{1}{(2 \pi)^{\frac{d}{2}} \sqrt{|\Sigma|}} \exp \left(-\frac{(x-\mu)^{T} \Sigma^{-1}(x-\mu)}{2}\right)
$$

if its curvature parameter $a_{i} \geq \varepsilon, p(x \mid \theta)$ is achieved by Equ. 7. $x_{T_{j}}$ is the selected points from the testing data $\mathbf{x}$ at the time instance of $T_{j}$, which can be achieved by Equ. 17.

$$
T_{j}=\mu_{f}-\frac{\eta_{j}\left(\mu_{f}-\mu_{f-1}\right)}{3}+\frac{\gamma_{j}\left(\mu_{f+1}-\mu_{f}\right)}{3} ;
$$

where $T_{j}$ is the time sampling points for the testing data; $j \in(1, \ldots, 5 \cdot k) ; \mu_{f}$ is the time label of the $f$ th component centre; $f=\lfloor(j-1) / 5\rfloor$; the parameters, $\eta$ and $\gamma$, are achieved by Equ. 18 and 19:

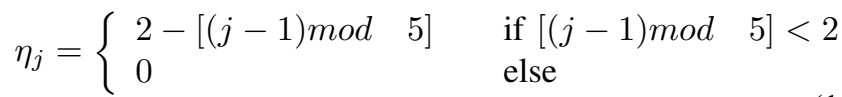

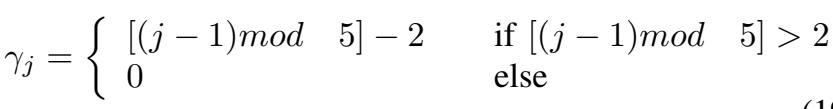

where mod is the modulo operation to find the remainder of division of one number by another. 
An example of the re-sampling process can be seen in Fig 9 where the re-sampled points are marked by the black star points from the testing data in the black line. The idea of resampling testing points is to reduce the computation cost and at the same time to maintain the accuracy of the likelihood. If the number of the points in the testing data $n>>k$, the re-sampling process can manage to reduce the number of the testing points to $5 k$, which can alleviate the computational burden for the recognition. Additionally, the re-sampling process selects the points according to the distribution of the components, which guarantees the re-sampled points cover the major distribution for the testing process.

\section{Experimental Results}

To evaluate the performance of FGMMs for classifying the EMG signals, FGMMs are compared with both traditional Gaussian Mixture Models (GMMs) and Support Vector Machine (SVM). To have a fare comparison, the recognition process for GMMs is the same as FGMMs which have been proposed in Sec. IV-C. The parameter for GMMs and FGMMs is the number of the component ranging from 2 to 20 with increments of one. As another popular machine learning method for classification [47], SVM use a kernel function to implicitly map the input vector into a high-dimensional space, and to maximise the margin between classes based on computational statistical theory. In this paper, radial basis function for the SVM classifier has been employed, which has been demonstrated with satisfactory performance in pattern recognition tasks [48], [39]. We used the one-against-all multiclass method for the multi-label classification, where for each label it builds a binary-class problem so instances associated with that label are in one class and the rest are in another class. The parameters for SVM are the kernel parameter ranging from 1 to 10 with increments of one and penalty cost whose range is from 1 to 501 with increments of 50 achieved by using LIBSVM [49] package. These parameters are selected with their best performance.

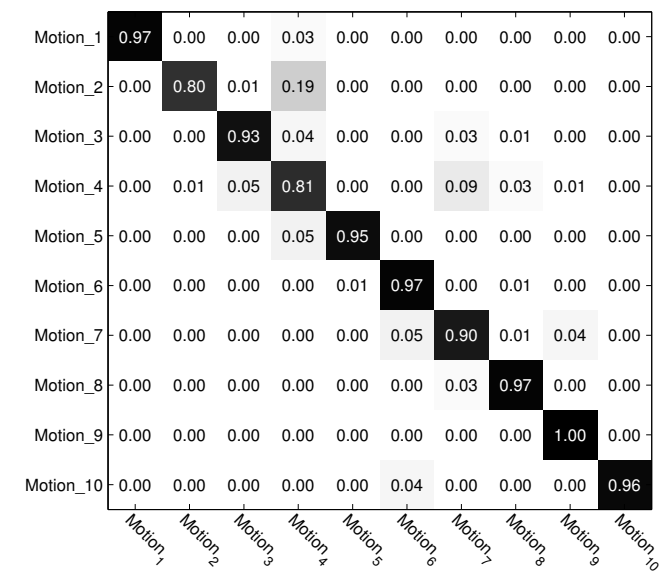

Fig. 10. Confusion Matrix for the ten hand motions using FGMMs, where the total accuracy is 92.75 percent

The performances of these three algorithms are evaluated by leave-one-subject-out cross-validation. Fig. 10 presents the confusion matrix for the ten hand motions in Sec. II-D using FGMMs. Among the 800 testing motions, FGMMs obtain 58 errors, and the total recognition rate is $92.75 \%$. Among the 10 motions, motion 9 has the full correct rate, and motions $1,6,8$ and 10 also receive high recognition rate of above 95 percent. However, the worst recognition rates are due to the misclassification of motions 2 and 4 with 20 and 19 percent error rates respectively.

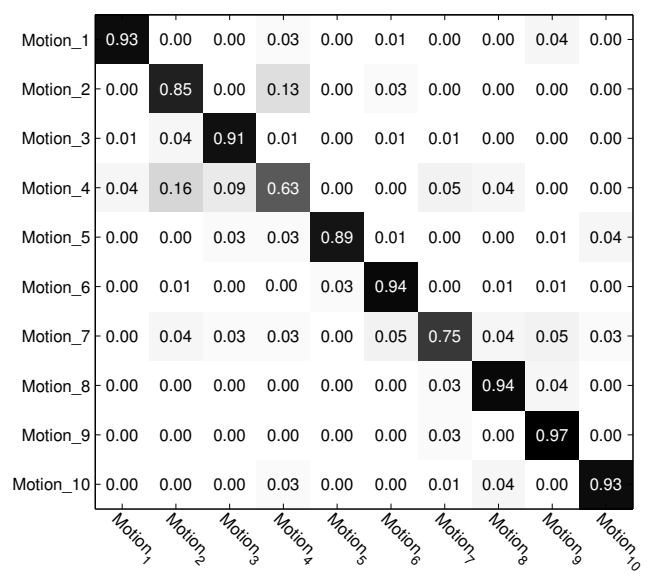

Fig. 11. Confusion Matrix for the ten hand motions using GMM, where the total accuracy is 87.25 percent

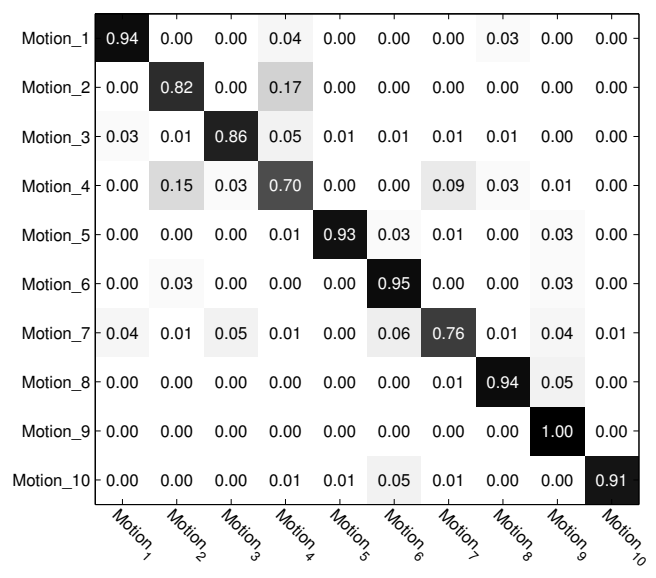

Fig. 12. Confusion Matrix for the ten hand motions using SVM, where the total accuracy is 88.13 percent

Fig. 11 and 12 show the recognition rates of GMMs and SVM respectively. The overall accuracy for GMMs is $87.25 \%$ and the one for SVM is $88.13 \%$. Motion 9 achieves high recognition rates with both GMMs and SVM methods, while motions 4 and 7 have high error rates with both GMMs and SVM methods. Compared with GMMs and SVM, FGMMs have the best overall performance, which reduces the error rate from $12.75 \%$ to $7.25 \%$, corresponding to a more than 40 percent error reduction. Motions 1, 3, 4, 5, 6, 7, 8 and 10 all have higher accuracies with FGMMs than with GMMs and SVM. Only motion 2 has the lowest accuracy with FGMMs than with GMMs and SVM. For all the methods, the motion 
9 always has a high accuracy rate, since this motion, lifting a cambelt, needs much more force and requires much stronger muscle contraction than others, which makes the EMG signals more identifiable than others.

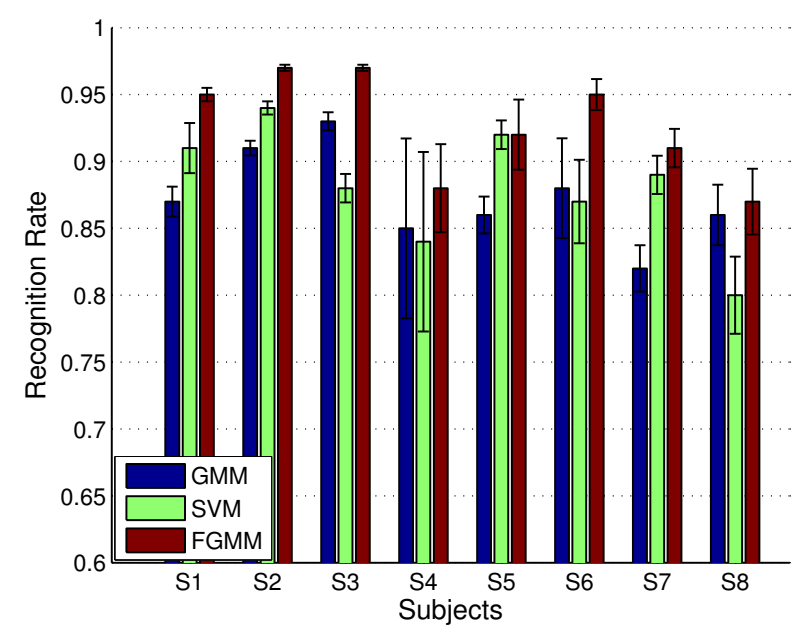

Fig. 13. Recognition results with means and variances of different subjects using different methods

On the other hand, Fig. 13 shows the average recognition rates and their variances of the eight subjects using different methods. For different subjects performing all motions, the recognition rates range from $80 \%$ to $97 \%$. The 2 nd subjects has the highest average accuracy rate of $94 \%$, while the subject eight has the lowest average rate of $84.33 \%$. From both the average accuracies and variances shown in Fig. 13, it concludes that FGMMs can reduce the error rates for all the subjects except the 5th subject, for whom the SVM has the highest accuracy while FGMMs have the second one. When achieving relatively high accuracy, e.g. subjects 1, 2 and 3, FGMMs have very small variances which are smaller than 0.02 . For the 6th subject, FGMMs have only five motions misclassified and it manages to improve the recognition rates of GMMs and SVM from around $87 \%$ to $95 \%$. Fig. 14 presents the box plot of different classifiers for the different subjects. Generally, FGMMs outperform GMMs and SVM for all the eight subjects in terms of the performance and the latter two have similar performances with each other.

\section{CONCluding Remarks}

In this paper, an integrated framework with multiple sensory information for analysing human hand motions has been proposed, providing effective solutions for motion capturing, data synchronisation and segmentation, correlation study of the sensory information and motion recognition. Three devices, i.e. CyberGlove, FingerTPS and Trigno wireless EMG sensors, have been integrated to simultaneously capture the finger angle trajectories, the contact forces and the forearm EMG signals at a fast sampling rate. An effective solution to automatically segment the manipulation primitives of different motions has been proposed using five-quick-grasp and four-quick-grasp protocols. Ten different grasps and in-hand manipulations from

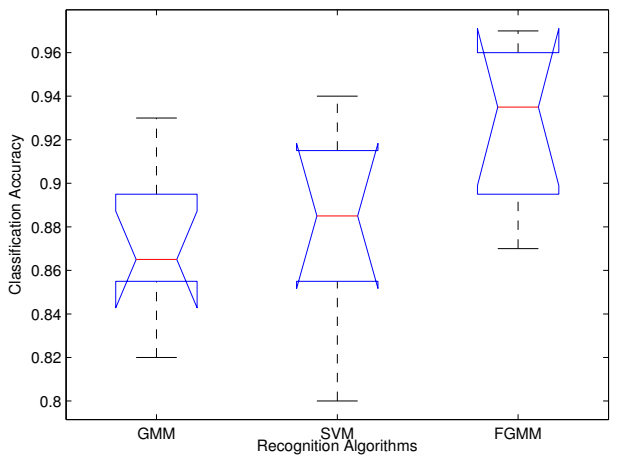

Fig. 14. Box plot results for the different classifiers for all subjects

eight different subjects have been analysed. In the knowledge base module, empirical copula has been employed to study the correlations of the different sensory information and the experimental results demonstrate there exist significant relationships between muscle signals and finger trajectories and between muscle signals and contact forces. In the motion recognition module, FGMMs have been used to recognise these ten motions from eight different subjects based on RMS features of the EMG signals and it achieved an overall 92.75 percent recognition rate, which is much higher than those of GMMs and SVM due to its nonlinear fitting capability. In terms of different subjects, FGMMs still outperformed the other two with improved accuracies.

The proposed framework integrates the state-of-the-art sensor technology, mature machine learning methods and signal processing algorithms. It provides a versatile and adaptable platform for researchers in robotics, biomedical engineering, $\mathrm{AI}$, and HCI to analyse human hand motions. The strong correlations among the signals indicate that the muscle signals can be potentially used to estimate the gesture or force of the hand motions. The main application of this framework is to control prosthetic hand via EMG signals. The proposed classification algorithms can effectively identify the amputees intended movements. The next step for controlling the prosthetic hand is to generate corresponding finger movements including the gestures and forces. The studied correlations in our framework provide a good reference for the further study to generate the desired trajectories. The future work is targeted to extend the knowledge base with human hand motion regressed primitives from the training motions and manipulation scenarios e.g object shape and contact points [50], and further to apply the framework into automatically controlling prosthetic hands such as the i-LIMB hand from Touch Bionics.

\section{REFERENCES}

[1] L. Dipietro, A. Sabatini, and P. Dario, "A survey of glove-based systems and their applications," IEEE Transactions on Systems, Man, and Cybernetics, Part C: Applications and Reviews, vol. 38, no. 4, pp. 461-482, 2008.

[2] C. Metcalf, S. Notley, P. Chappell, J. Burridge, and V. Yule, "Validation and application of a computational model for wrist and hand movements using surface markers," IEEE Transactions on Biomedical Engineering, vol. 55, no. 3, pp. 1199-1210, 2008. 
[3] Z. Ju and H. Liu, "A unified fuzzy framework for human hand motion recognition," IEEE Transactions on Fuzzy Systems, vol. 19, no. 5, pp. 901-913, 2011.

[4] T. Moeslund and E. Granum, "A survey of computer vision-based human motion capture," Computer Vision and Image Understanding, vol. 81, no. 3, pp. 231-268, 2001

[5] A. Malinowski and H. Yu, "Comparison of embedded system design for industrial applications," IEEE Transactions on Industrial Informatics, vol. 7, no. 2, pp. 244-254, 2011.

[6] X. Ji and H. Liu, "Advances in view-invariant human motion analysis: A review," IEEE Transactions on Systems, Man, and Cybernetics, Part C: Applications and Reviews, vol. 40, no. 1, pp. 13-24, 2010.

[7] Z. Ren, J. Meng, J. Yuan, and Z. Zhang, "Robust hand gesture recognition with kinect sensor," in Proceedings of the 19th ACM international conference on Multimedia, pp. 759-760, ACM, 2011.

[8] Z. Zafrulla, H. Brashear, T. Starner, H. Hamilton, and P. Presti, "American sign language recognition with the kinect," in Proceedings of the 13 th international conference on multimodal interfaces, pp. 279-286, ACM, 2011.

[9] S. Calinon, P. Evrard, E. Gribovskaya, A. Billard, and A. Kheddar, "Learning collaborative manipulation tasks by demonstration using a haptic interface," in International Conference on Advanced Robotics, pp. 1-6, IEEE, 2009.

[10] S. Saliceti, J. Ortiz, A. Cardellino, L. Rossi, and J. Fontaine, "Fusion of tactile sensing and haptic feedback for unknown object identification aimed to tele-manipulation," in IEEE Conference on Multisensor Fusion and Integration for Intelligent Systems, pp. 205-210, IEEE, 2010.

[11] T. Takaki and T. Omata, "High-performance anthropomorphic robot hand with grasping-force-magnification mechanism," IEEE/ASME Transactions on Mechatronics, vol. 16, no. 3, p. 583, 2011.

[12] M. Ferre, I. Galiana, R. Wirz, and N. Tuttle, "Haptic device for capturing and simulating hand manipulation rehabilitation," IEEE/ASME Transactions on Mechatronics, vol. 16, no. 5, pp. 808-815, 2011.

[13] C. Park, J. Yoo, and A. Howard, "Transfer of skills between human operators through haptic training with robot coordination," in IEEE International Conference on Robotics and Automation, pp. 229-235, IEEE, 2010.

[14] T. Saponas, D. Tan, D. Morris, R. Balakrishnan, J. Turner, and J. Landay, "Enabling always-available input with muscle-computer interfaces," in Proceedings of the 22nd annual ACM symposium on User interface software and technology, pp. 167-176, ACM, 2009.

[15] X. Tang, Y. Liu, C. Lv, and D. Sun, "Hand motion classification using a multi-channel surface electromyography sensor," Sensors, vol. 12, no. 2 , pp. 1130-1147, 2012.

[16] C. Cipriani, F. Zaccone, S. Micera, and M. Carrozza, "On the shared control of an emg-controlled prosthetic hand: analysis of user-prosthesis interaction," IEEE Transactions on Robotics, vol. 24, no. 1, pp. 170-184, 2008.

[17] C. Castellini and P. van der Smagt, "Surface emg in advanced hand prosthetics," Biological cybernetics, vol. 100, no. 1, pp. 35-47, 2009.

[18] D. Zhang, X. Chen, S. Li, P. Hu, and X. Zhu, "Emg controlled multifunctional prosthetic hand: Preliminary clinical study and experimental demonstration," in IEEE International Conference on Robotics and Automation, pp. 4670-4675, IEEE, 2011.

[19] M. Williams and R. Kirsch, "Evaluation of head orientation and neck muscle emg signals as command inputs to a human-computer interface for individuals with high tetraplegia," IEEE Transactions on Neural Systems and Rehabilitation Engineering, vol. 16, no. 5, pp. 485-496, 2008.

[20] A. Usakli, S. Gurkan, F. Aloise, G. Vecchiato, and F. Babiloni, "On the use of electrooculogram for efficient human computer interfaces," Computational Intelligence and Neuroscience, vol. 2010, pp. 1-5, 2010.

[21] P. Langhorne, F. Coupar, and A. Pollock, "Motor recovery after stroke: a systematic review," The Lancet Neurology, vol. 8, no. 8, pp. 741-754, 2009.

[22] N. Ho, K. Tong, X. Hu, K. Fung, X. Wei, W. Rong, and E. Susanto, "An emg-driven exoskeleton hand robotic training device on chronic stroke subjects: Task training system for stroke rehabilitation," in IEEE International Conference on Rehabilitation Robotics, pp. 1-5, IEEE, 2011.

[23] M. Ceruti, V. Dinh, N. Tran, H. Van Phan, L. Duffy, T. Ton, G. Leonard, E. Medina, O. Amezcua, S. Fugate, et al., "Wireless communication glove apparatus for motion tracking, gesture recognition, data transmission, and reception in extreme environments," in Proceedings of the 2009 ACM symposium on Applied Computing, pp. 172-176, ACM, 2009.
[24] N. Hendrich, D. Klimentjew, and J. Zhang, "Multi-sensor based segmentation of human manipulation tasks," in IEEE Conference on Multisensor Fusion and Integration for Intelligent Systems, pp. 223-229, IEEE, 2010.

[25] J. Romano, S. Gray, N. Jacobs, and K. Kuchenbecker, "Toward tactilely transparent gloves: Collocated slip sensing and vibrotactile actuation," in EuroHaptics conference and Symposium on Haptic Interfaces for Virtual Environment and Teleoperator Systems. World Haptics 2009. Third Joint, pp. 279-284, Ieee, 2009.

[26] D. Yang, J. Zhao, Y. Gu, L. Jiang, and H. Liu, "Estimation of hand grasp force based on forearm surface emg," in International Conference on Mechatronics and Automation, pp. 1795-1799, IEEE, 2009.

[27] H. Liu, "Exploring human hand capabilities into embedded multifingered object manipulation," IEEE Transactions on Industrial Informatics, vol. 7, no. 3, pp. 389-398, 2011.

[28] Z. Ju and H. Liu, "A generalised framework for analysing human hand motions based on multisensor information," in IEEE International Conference on Fuzzy Systems, pp. 1 -6, june 2012.

[29] T. Yoshikawa, "Multifingered robot hands: Control for grasping and manipulation," Annual Reviews in Control, vol. 34, no. 2, pp. 199-208, 2010.

[30] C. Spearman, "The proof and measurement of association between two things," The American journal of psychology, vol. 15, no. 1, pp. 72-101, 1904.

[31] E. Wolff, "N-dimensional measures of dependence.," Stochastica: revista de matemática pura y aplicada, vol. 4, no. 3, pp. 175-188, 1980.

[32] F. Schmid and R. Schmidt, "Multivariate conditional versions of spearman's rho and related measures of tail dependence," Journal of multivariate analysis, vol. 98, no. 6, pp. 1123-1140, 2007.

[33] R. Nelsen, "An Introduction to Copulas (Springer Series in Statistics)," 2006.

[34] A. Sklar, "Fonctions de répartition a n dimensions et leurs marges.," Publ Inst Statist Univ Paris, vol. 8, pp. 229-231, 1959.

[35] W. Kruskal, "Ordinal measures of association," Journal of the American Statistical Association, vol. 53, no. 284, pp. 814-861, 1958.

[36] E. Lehmann, "Some concepts of dependence," Ann. Math. Statist, vol. 37, no. 1, pp. 137-1, 1966.

[37] M. Yewale and M. Bharne, "Artificial neural network approach for hand gesture recognition," International Journal of Engineering Science and Technology (IJEST), vol. 3, no. 4, pp. 2603-2608, 2011.

[38] D. Martinez and D. Kragic, "Modeling and recognition of actions through motor primitives," in IEEE International Conference on Robotics and Automation (ICRA08), pp. 1704-1709, 2008.

[39] X. Chen, X. Zhu, and D. Zhang, "A discriminant bispectrum feature for surface electromyogram signal classification," Medical engineering \& physics, vol. 32, no. 2, pp. 126-135, 2010.

[40] B. Bedregal, A. Costa, and G. Dimuro, "Fuzzy rule-based hand gesture recognition," in Artificial Intelligence in Theory and Practice, vol. 217 of IFIP International Federation for Information Processing, pp. 285-294, Springer Boston, 2006.

[41] Z. Ju, H. Liu, X. Zhu, and Y. Xiong, "Dynamic Grasp Recognition Using Time Clustering, Gaussian Mixture Models and Hidden Markov Models," Journal of Advanced Robotics, vol. 23, pp. 1359-1371, 2009.

[42] S. Ba and J. Odobez, "Recognizing visual focus of attention from head pose in natural meetings," IEEE Transactions on Systems, Man, and Cybernetics, Part B: Cybernetics, vol. 39, no. 1, pp. 16-33, 2009.

[43] Y. Huang, K. Englehart, B. Hudgins, and A. Chan, "A gaussian mixture model based classification scheme for myoelectric control of powered upper limb prostheses," IEEE Transactions on Biomedical Engineering, vol. 52, no. 11, pp. 1801-1811, 2005.

[44] L. Lu, A. Ghoshal, and S. Renals, "Regularized subspace gaussian mixture models for speech recognition," IEEE Signal Processing Letters, vol. 18, pp. 419-422, 2011.

[45] Z. Ju and H. Liu, "Fuzzy gaussian mixture models," Pattern Recognition, vol. 45, no. 3, pp. 1146-1158, 2012.

[46] A. Phinyomark, C. Limsakul, and P. Phukpattaranont, "Emg feature extraction for tolerance of $50 \mathrm{hz}$ interference," in Proc. of PSU-UNS Inter. Conf. on Engineering Technologies, ICET, pp. 289-293, 2009.

[47] V. Vapnik, The nature of statistical learning theory. Springer Verlag, 2000.

[48] I. Steinwart and A. Christmann, Support vector machines. Springer Verlag, 2008.

[49] C.-C. Chang and C.-J. Lin, "Libsvm: A library for support vector machines," ACM Trans. Intell. Syst. Technol., vol. 2, pp. 27:1-27:27, May 2011.

[50] Q. Le, D. Kamm, A. Kara, and A. Ng, "Learning to grasp objects with multiple contact points," in IEEE International Conference on Robotics and Automation, pp. 5062-5069, 2010. 


\section{APPENDIX}

\section{A. Projected points}

As shown in Fig. 15, supposing $z=\left[z_{1 j}, z_{2 j}\right]^{T}$ is the $j$ th projective point of the sample $\left[v_{1 t}, v_{2 t}\right]$, the arc length of $z$ is formulated as

$$
l_{j}\left(v_{1 t}\right)=\int_{(0, b)}^{\left(z_{1 j}, z_{2 j}\right)} \sqrt{\left(d z_{1 j}\right)^{2}+\left(d z_{2 j}\right)^{2}}
$$

$z$ is on the standard curve, so it satisfies $z_{2}=a z_{1}^{2}+b$. So we have

$$
\begin{aligned}
& l_{j}\left(v_{1 t}\right)=\frac{1}{2} z_{1 j} \sqrt{1+4 a^{2} z_{1 j}^{2}} \\
& +\frac{1}{4|a|} \ln \left(2|a| z_{1 j}+\sqrt{1+4 a^{2} z_{1 j}^{2}}\right)
\end{aligned}
$$

The distance between the sample $\left[v_{1 t}, v_{2 t}\right]^{T}$ and its projective point $z$ is

$$
l_{j}\left(v_{2 t}\right)=\sqrt{\left(v_{1 t}-z_{1 j}\right)^{2}+\left(v_{2 t}-z_{2 j}\right)^{2}}
$$

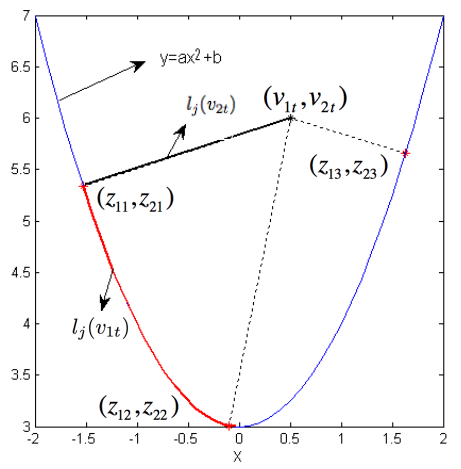

Fig. 15. Projective points. The point $\left(z_{1 t}, z_{2 t}\right)$ has three projected points, which are marked in red star. The red curve illustrates the arc length of the projected point $\left(z_{11}, z_{21}\right.$ and the black line gives the distance between the point $\left(z_{1 t}, z_{2 t}\right)$ and projected point $\left(z_{11}, z_{21}\right)$

\section{B. EM Algorithm}

The EM algorithm of FGMMs is shown in Algorithm 1.

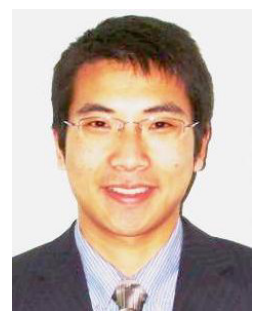

Zhaojie Ju (M'08) received the B.S. in automatic control and the M.S. in intelligent robotics both from Huazhong University of Science and Technology, China, in 2005 and 2007 respectively, and the Ph.D. degree in intelligent robotics at the University of Portsmouth, UK, in 2010.

$\mathrm{Dr} \mathrm{Ju}$ is currently a Lecturer in the School of Creative Technologies, University of Portsmouth, UK. He previously held research appointments in the Department of Computer Science, University College London and Intelligent Systems and Biomedical Robotics group, University of Portsmouth, UK. His research interests are in machine intelligence, robot learning and pattern recognition. He has been awarded one Best Paper Awards, one Best Competition Paper Award, and one Travel Grant Award.
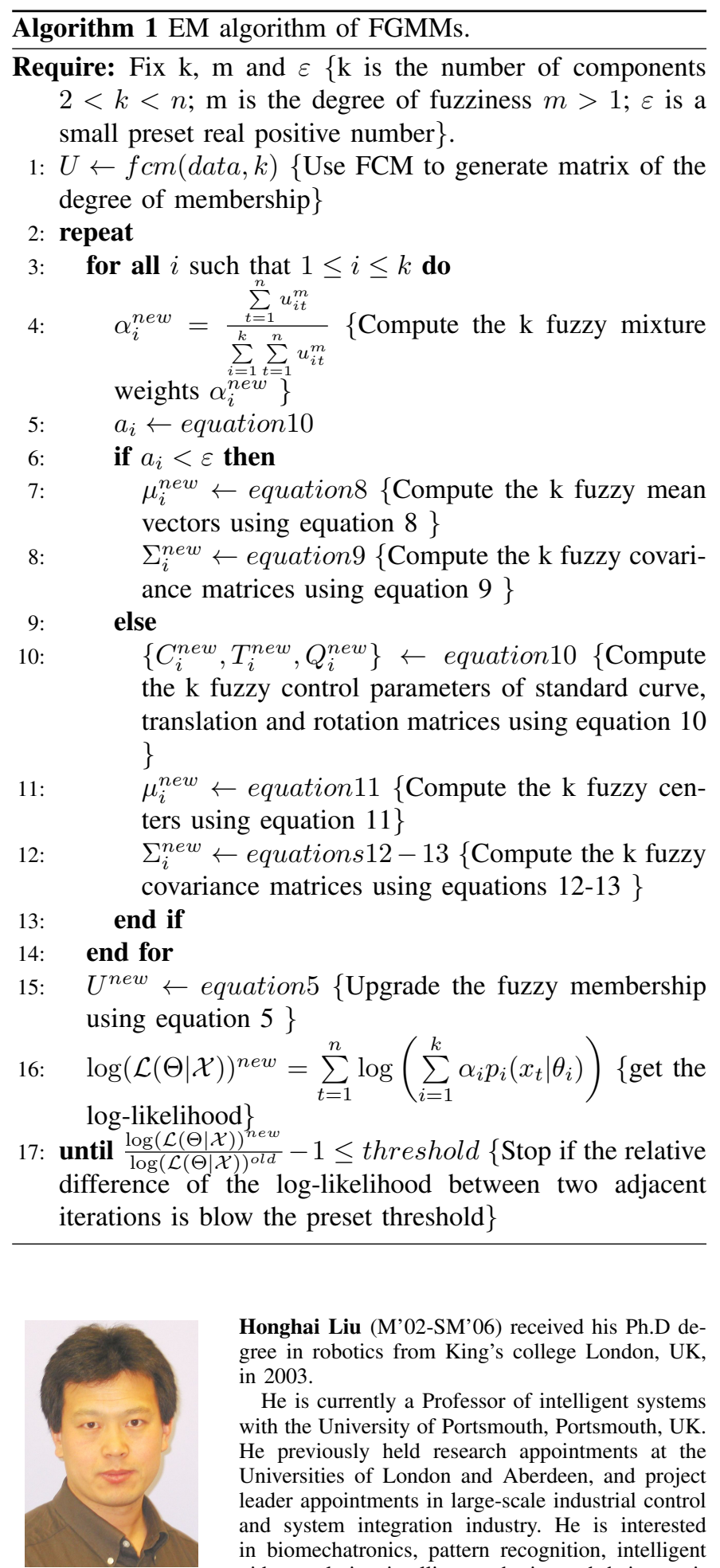

Honghai Liu (M'02-SM'06) received his Ph.D degree in robotics from King's college London, UK, in 2003.

$\mathrm{He}$ is currently a Professor of intelligent systems with the University of Portsmouth, Portsmouth, UK. He previously held research appointments at the Universities of London and Aberdeen, and project leader appointments in large-scale industrial control and system integration industry. $\mathrm{He}$ is interested in biomechatronics, pattern recognition, intelligent video analytics, intelligent robotics and their practical applications with an emphasis on approaches that could make contribution to the intelligent connection of perception to action using contextual information. He is Associate Editor of IEEE Transactions on Industrial Informatics, IEEE Transactions on Fuzzy Systems, IEEE Transactions on Human-Machine Systems.

Dr Liu is a Fellow of the Institution of Engineering and Technology. 